\title{
Modified Three-Dimensional Multicarrier Optical Prime Codes
}

\author{
Rajesh Yadav and Gurjit Kaur \\ Department of ECE, School of ICT, Gautam Buddha University, Greater Noida, India \\ Correspondence should be addressed to Rajesh Yadav; raj_opyadav@yahoo.co.in
}

Received 20 May 2016; Revised 26 July 2016; Accepted 22 August 2016

Academic Editor: Gang-Ding Peng

Copyright (C) 2016 R. Yadav and G. Kaur. This is an open access article distributed under the Creative Commons Attribution License, which permits unrestricted use, distribution, and reproduction in any medium, provided the original work is properly cited.

\begin{abstract}
We propose a mathematical model for novel three-dimensional multicarrier optical codes in terms of wavelength/time/space based on the prime sequence algorithm. The proposed model has been extensively simulated on MATLAB for prime numbers $(P)$ to analyze the performance of code in terms of autocorrelation and cross-correlation. The simulated outcome resembles the mathematical model and gives better results over other methods available in the literature as far as autocorrelation and crosscorrelation are concerned. The proposed 3D optical codes are more efficient in terms of cardinality, improved security, and providing quality of services.
\end{abstract}

\section{Introduction}

Optical Code Division Multiple Access (OCDMA) network has a great potential to cater the needs of the fast access communication system. OCDMA is a suitable multiple access technique for local area network where traffic is bursty in nature [1]. Optical CDMA is an efficient multiplexing scheme, as it does not require synchronization between the transmitter and receiver and there is the efficient utilization of available bandwidth [2]. The performance of optical CDMA networks depends on the optimal selection of the optical code and coding configuration. It is desired to have code set that can correctly decode the desired user signal in the presence of other users [3]. The motivation for designing more dimensional code is to have increased code set and also have significant code weight to support quality of services for the given code length. The length of a code plays an important role in system performance and system complexity [4], although it is possible to improve the correlation property by using long code words. However, the code length cannot be increased much because of the power and processing time restrictions for encoder and decoder [5]. The weight of code represents the signal power. A code sequence with larger code weight is less sensitive to interference than that with a lower code weight. In this paper, novel modified threedimensional (3D) multicarrier prime codes are proposed having sufficient code weight along with significant code length for supporting QoS in optical CDMA communication systems. The construction of proposed three-dimensional codes is conceptualized on the basis of prime sequence algorithm.

The rest of paper is organized as follows: Section 2 discusses the OCDMA coding theory that gives an insight of the optical codes for optical CDMA communication system. A concise introduction to optical prime codes for OCDMA systems is also discussed. Section 3 presents the mathematical modeling of proposed wavelength/time/space modified 3D multicarrier optical prime codes. Section 4 discusses the result of the proposed 3D optical prime codes and its performance estimation in terms of the autocorrelation and cross-correlation function. In Section 5 the current findings along with the future directions are concluded. The paper ends with the references studied and cited in the paper.

\section{Optical Codes}

A lot of researchers have studied optical codes like onedimensional (1D), two-dimensional (2D), and now threedimensional codes to provide efficient codes. Prucnal has proposed one-dimensional optical code based on the prime number $P$ over a Galois field $\operatorname{GF}(P)$. The codes have an autocorrelation value of $P-1$ and cross-correlation value of two [6]. The construction of prime codes is based on the congruence codes. Later on, one-dimensional prime code 


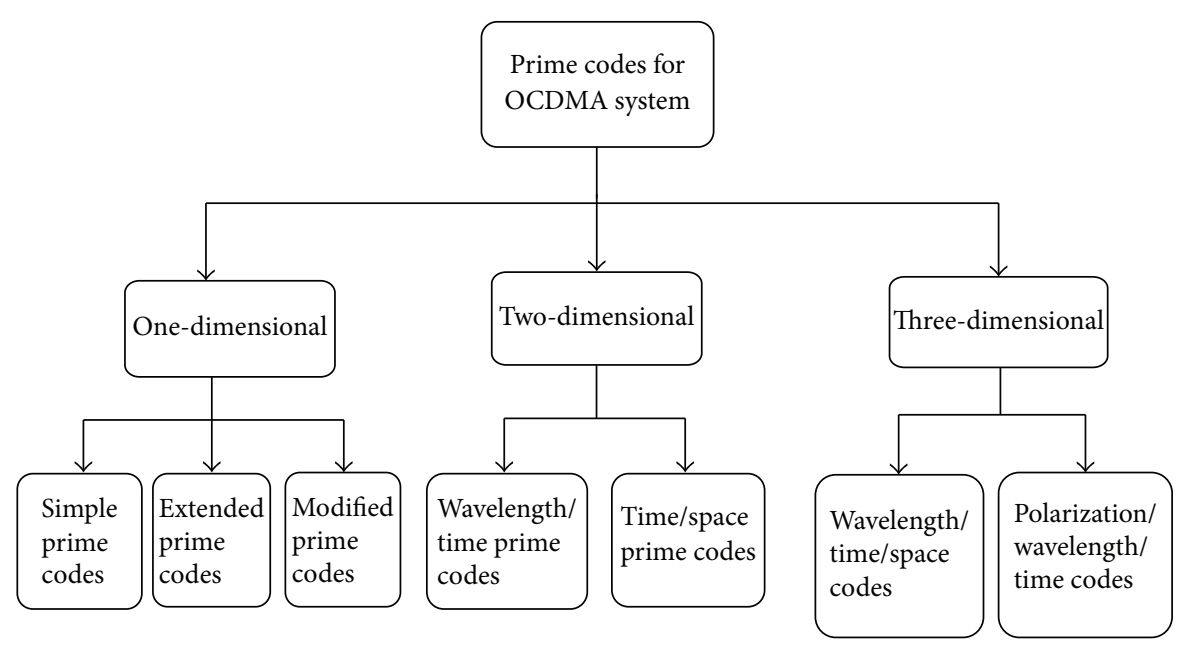

Figure 1: Prime code word family hierarchy [11].

with improved correlation property is proposed by many researchers [7-10]. In the one-dimensional code to increase the code set size, the code length is increased significantly to keep the correlation property satisfactory. To overcome this limitation two-dimensional codes are developed with better code set size and good correlation properties. Mendez et al. describe the 2D codes constructed from $1 \mathrm{D}$ code based on Golomb ruler to increase the number of code set sizes [7]. $\mathrm{Yu}$ and Park have proposed an algorithm to construct 2D code based on 1D prime sequence [8]. Yim et al. proposed a design for wavelength/time 2D code for O-CDMA and carried out the performance analysis of the 2D codes [9]. The performance analysis of the two-dimensional optical codes for bit error rate and a number of users supported carried out by Shivaleela and Kaur has revealed that there is a significant improvement in optical CDMA system using 2D codes over $1 \mathrm{D}$ code $[10,11]$.

The successful performance of two-dimensional optical codes over the one-dimensional codes has led the researcher to construct the three-dimensional codes with better code set size and improved system performance. McGeehan presented a time-wavelength-polarization 3D optical code for increasing the number of users in OCDMA LAN's application. This coding technique has limitation due to the polarization mode dispersion and complex polarization control at all the stages in the network $[12,13]$. Kumar et al. present multiple pulse per plane codes using a row-wise orthogonal pairs (RWOP) algorithm for wavelength and spatial channel allocation [14]. The design offers the very low probability of error due to multiple access interference at lower cardinality. The RWOP algorithm based 3D code has a limitation imposed by multipath interference. The hierarchy of prime sequence codes is represented in Figure 1 [11]. Here we proposed wavelength/time/space spread 3D optical codes based on prime sequence algorithm derived from multicarrier prime codes for OCDMA $[14,15]$. The comparative analysis of $1 \mathrm{D}$, $2 \mathrm{D}$, and $3 \mathrm{D}$ optical codes based on prime sequence is carried out by Yadav and Kaur [16]. The generation algorithm of 3D codes is discussed in detail and the orthogonality of the $3 \mathrm{D}$ optical codes is demonstrated mathematically and simulated on the MATLAB.

\section{Generation of 3D Optical Code}

In this research work, we have presented new modified threedimensional multicarrier optical code for OCDMA network based on prime sequence algorithm. The $3 \mathrm{D}$ codes family can be represented as $\left(W * T * S, w, \lambda_{a}, \lambda_{c}\right)$ where $W, T$, and $S$ denote the number of wavelengths, time, and spatial channels domain used, respectively. " $w$ " signifies the weight of the code. $\lambda_{a}$ and $\lambda_{c}$ are the autocorrelation and cross-correlation of the optical code, respectively. Figure 2 illustrates the algorithm of modified three-dimensional multicarrier prime code constructions.

Three-dimensional optical code construction starts with the prime number $P$ over the Galois field (GF). A prime sequence is constructed for a prime number $P$ as $[6,17]$

$$
\begin{aligned}
& Z_{r}=\left(z_{r, 0}, z_{r, 1}, \ldots, z_{r, s}, \ldots, z_{r,(P-1)}\right) \\
& r=0,1, \ldots, P-1,
\end{aligned}
$$

where the element in this prime sequence is given by

$$
Z_{r, s}=r \cdot s \quad(\bmod P)
$$

where $r=(0,1, \ldots, P-1)$ and $s=(0,1, \ldots, P-1)$ are the elements over the Galois field $\mathrm{GF}(P)$. For prime number $P=3$, the corresponding code set matrix $Z_{r, s}$ is shown by (3), where $r$ and $s$ are the elements over the Galois field GF(3). The following $3 \times 3$ matrix will be constructed:

$$
Z_{r, s}=\left[\begin{array}{ccc}
Z_{0,0} & Z_{0,1} & Z_{0,2} \\
Z_{1,0} & Z_{1,1} & Z_{1,2} \\
Z_{2,0} & Z_{2,1} & Z_{2,2}
\end{array}\right] .
$$


This matrix represents a code set for prime number $P=3$. In this $3 \mathrm{D}$ prime code set, every code word is represented by a matrix as given by

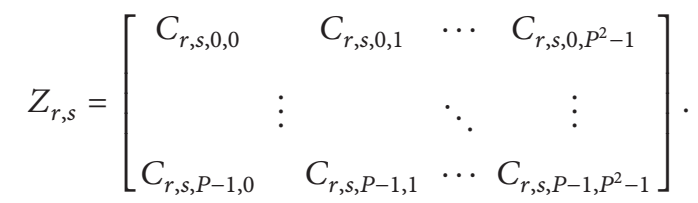

This matrix represents a code word whose element can be derived as $C_{m, k}=C_{r, s, m, 0} \cdots C_{r, s, m, k} \cdots C_{r, s, m, P^{2}-1}$, where $r=(0,1, \ldots, P-1), s=(0,1, \ldots, P-1), m=$ $(0,1, \ldots, P-1)$, and $k=\left(0,1, \ldots, P^{2}-1\right)$. With the prime sequence matrix $Z_{r, s}$ the value of $C_{m, k}$ will be mapped as

$$
C_{m, k}= \begin{cases}W_{r \cdot n \oplus t \cdot s \cdot m, r \cdot m \oplus s \cdot n} & \text { if } k=((r \cdot n \oplus t \cdot s \cdot m) P+n), \text { for } m, n=\{0,1, \ldots, P-1\} \\ 0 & \text { elsewere. }\end{cases}
$$

This mapping will define the value of matrix elements or position of wavelength in the code word matrix of size $3 \times 9$ for $P=3$. A matrix is constructed by setting each element according to the rule $C_{m, k}=W_{r \cdot n \oplus t \cdot s \cdot m, r \cdot m \oplus s \cdot n}$, where $n=(0,1, \ldots, P-1), m=(0,1, \ldots, P-1), t=$ $\{0,1, \ldots, P-1\} /[B]$, and $\oplus$ denotes a modulo addition. $[B]$ is a set of numbers that contains every integer designed with $1 / s^{2}(\bmod P)$ for $s=\{1,2, \ldots, P-1\} / 2$. The genuine value of $t$ can be calculated using this formula and that can be used to obtain $Z_{r, s}$ for various prime numbers. Each element $W_{r \cdot n \oplus t \cdot s \cdot m, r \cdot m \oplus s \cdot n}$ represents a single transmitting wavelength, among $P^{2}$ different wavelengths in the $m$ th space slot and $n$th time period position in $Z_{r, s}$. A binary $3 \mathrm{D}$ multicarrier prime code can be derived as having the exact position of wavelength in the space slot and time period.

In $Z_{r, s}$ code word matrix, each row will have one set of disjoint wavelength and in total, there are $P$ distinct wavelengths per row. The space group is represented by $m=$ $(0,1, \ldots, P-1)$. In this way, we have $P^{2}-1$ prime code words of length $P^{2}$ and weight $P^{2}$ and have $P^{2}$ different wavelengths where $m \leq P$. These $P^{2}$ distinct wavelengths are divided into " $m$ " space groups with $P$ different wavelengths per space/channel. Every time slot can have a minimum of one wavelength. The optical code words can then be transmitted over " $m$ " different channels having $P$ different wavelengths each distributed over $P^{2}$ time slots thus acting as a 3D code. In this way, the position of $P$ different wavelengths can be calculated in every $m$ spatial channel according to the formula given in (5). The cardinality of $3 \mathrm{D}$ optical prime code is given by $|C|=P^{2}-1$; therefore for $P=3$ it will be 8 and $Z_{0,0}$ for $(a, b)=(0,0)$, respectively, will not exist.

For prime number $P=3$ the valid value of $t$ we have is 2. In the codeword $Z_{0,1}, r=0$ and $s=1$ are in GF(3). The elements of the code word matrix are calculated according to the rule $C_{m, k}=W_{r \cdot n \oplus t \cdot s \cdot m, r \cdot m \oplus s \cdot n}$ as shown in

$$
Z_{0,1}=\left[\begin{array}{ccc}
W_{0,0} W_{0,1} W_{0,2} & 000 & 000 \\
000 & 000 & W_{2,0} W_{2,1} W_{2,2} \\
000 & W_{1,0} W_{1,1} W_{1,2} & 000
\end{array}\right]
$$

The corresponding code matrix is of size $3 \times 9$ and weight 9. Each " $W$ " in the code word matrix represents the position of " 1 " in the code word that will be transmitted using an optical pulse of certain wavelength over the space channel in the corresponding time slot. It can be seen that each code word matrix of a three-dimensional code is divided into $P$ rows in the space domain having length $P^{2}$ for each row in the time domain; the $P^{2}$ column has been divided into $P$ groups each having $P$ subcolumns.

The code word has $P^{2}$ wavelengths distributed over the $P^{2}$ time slot with each column having only one wavelength and each space channel will have $P$ wavelengths. The $P^{2}$ wavelengths are in the shape of 3 groups and 3 wavelengths per group. The length of the 3D optical code word is 9 for $P=3$. Figure 3 shows the visualization of a wavelength/time/space spread $3 \mathrm{D}$ code word $Z_{0,1}$ for prime sequence $(P=3)$ and $t=2$. Similarly, for the code word $Z_{2,2}$ the elements are calculated according to the rule $C_{m, k}=W_{r \cdot m \oplus t \cdot s \cdot n, r \cdot n \oplus s \cdot m}$. The corresponding code matrices $Z_{r, s}$ for $r=2$ and $s=$ 2 are of size $3 \times 9$ and weight 9 as shown in Figure 4 . Equation (7) shows the resulting code word matrix as follows:

$$
Z_{2,2}=\left[\begin{array}{lll}
W_{0,0} 00 & 00 W_{1,1} & 0 W_{2,2} 0 \\
0 W_{0,1} 0 & W_{1,2} 00 & 00 W_{2,0} \\
00 W_{0,2} & 0 W_{1,0} 0 & W_{2,1} 00
\end{array}\right]
$$

In OCDMA system using modified 3D multicarrier prime code, each subscriber is allocated a code matrix as its code word. To transmit information bit "1" subscriber sends out a sequence of the optical signal in the space, time, and wavelength domain as per the intended receiver code word matrix. At the receiving end, the optical signal in every spatial channel is segregated and routed to correlate the every wavelength separately in the time domain. After successfully correlating the received optical signals are combined together, thus resulting in a high peak optical signal for the proposed receiver. This is considered as autocorrelation function, whereas for all others, it gives a series of low peak optical signal. This is considered as cross-correlation function. The received peak optical pulse is further passed 


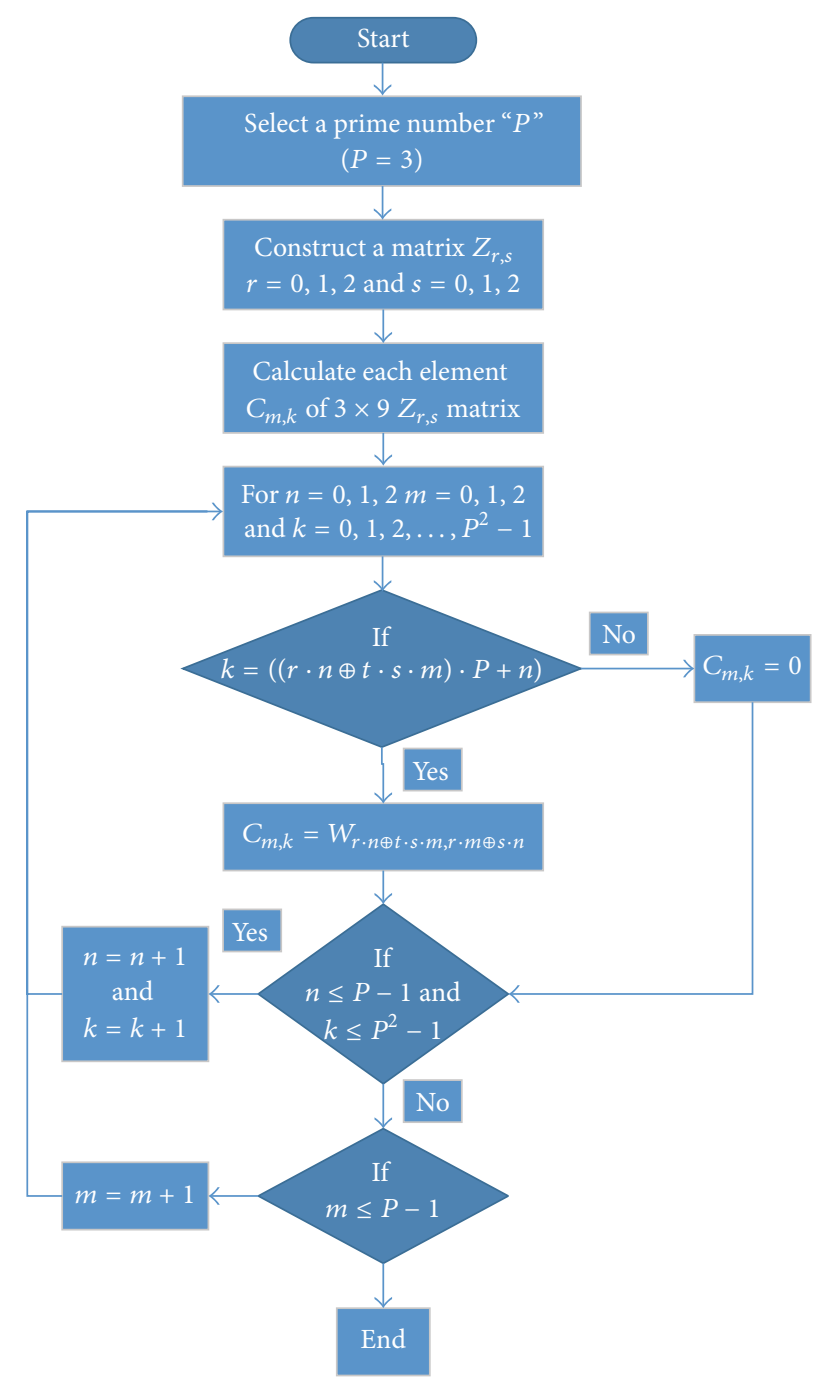

FIGURE 2: Flowchart of three-dimensional code construction algorithm.

\begin{tabular}{|c|c|c|c|c|c|c|c|c|c|c|}
\hline & $m=2$ & $W_{00}$ & $W_{01}$ & $W_{02}$ & 0 & 0 & 0 & 0 & 0 & 0 \\
\hline & $m=1$ & 0 & 0 & 0 & 0 & 0 & 0 & $W_{20}$ & $W_{21}$ & $W_{22}$ \\
\hline & $m=0$ & 0 & 0 & 0 & $W_{10}$ & $W_{11}$ & $W_{12}$ & 0 & 0 & 0 \\
\hline
\end{tabular}

Figure 3: Visualization of $3 \mathrm{D}$ optical prime code word $Z_{0,1}$ over $\mathrm{GF}(P=3)$ and $t=2$.

to photodetector to detect the bit " 1. . For transmitting information bit " 0 " nothing is sent.

\section{Result and Discussion}

From optical CDMA network point of view, there is a need to design more secure codes with sufficient code weight, optimal code length, and higher code set size. The proposed code is compared with the $1 \mathrm{D}$ and $2 \mathrm{D}$ optical codes based on prime sequence algorithm for analysis as presented in Figure 5. It

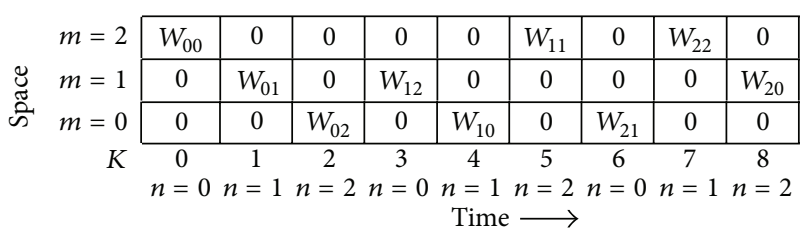

FIGURE 4: Visualization of 3D optical prime code word $Z_{2,2}$ over $\mathrm{GF}(P=3)$ and $t=2$.

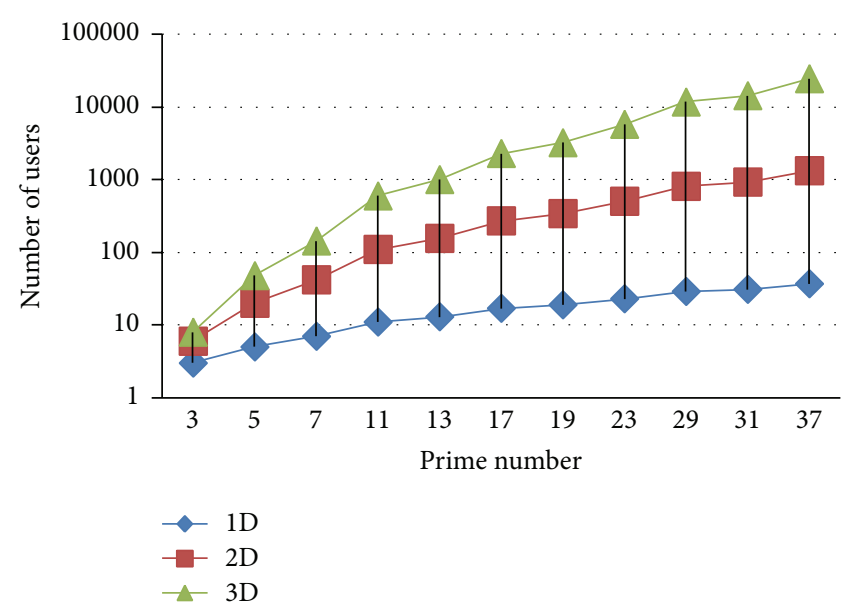

FIGURE 5: Number of users versus prime number for different optical prime codes.

can be observed that the proposed 3D codes are better in terms of a number of available codes (Cardinality) whereas they have comparable code length for a given prime number. However, the proposed $3 \mathrm{D}$ codes have higher code weight than $1 \mathrm{D}$ and $2 \mathrm{D}$ codes.

In optical CDMA, the interference among the users sharing the common fiber channel known as the multiple access interference is usually the dominant source of bit errors [6]. The performance of optical codes in OCDMA networks is mainly measured in terms of the autocorrelation and cross-correlation function values. The cross-correlation value depicts the interference between users. In contrast, the autocorrelation value depicts signal power and differentiates the users in the presence of other users. The intelligent design of the code word sequence is important to reduce the contribution of MAI to the total received signal. The address code words must satisfy two conditions; that is, all address code words should be distinguishable from shifted versions of it and all address code words should be distinguished from a possibly shifted version of every other code word in a code set [16].

The autocorrelation and cross-correlation value of the developed 3D optical code are evaluated on MATLAB tool. The wavelength/time/space spread 3D optical code is represented by $\left(W * T * S, w, \lambda_{a}, \lambda_{c}\right)$. "W" signifies the wavelengths, " $T$ " denotes the time slots, and " $S$ " specifies the space channels. " $w$ " signifies the weight of the code. $\lambda_{a}$ and $\lambda_{c}$ are the autocorrelation and cross-correlation of the code, 


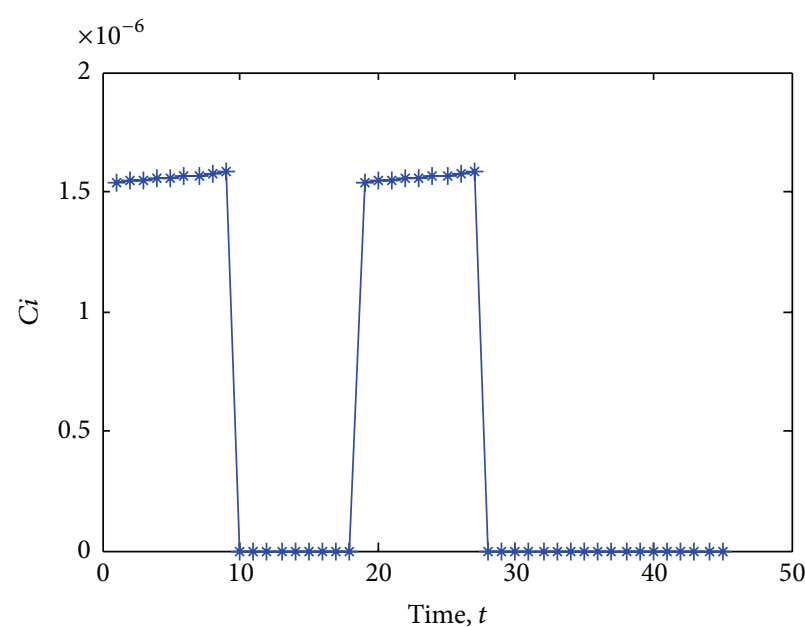

(a)

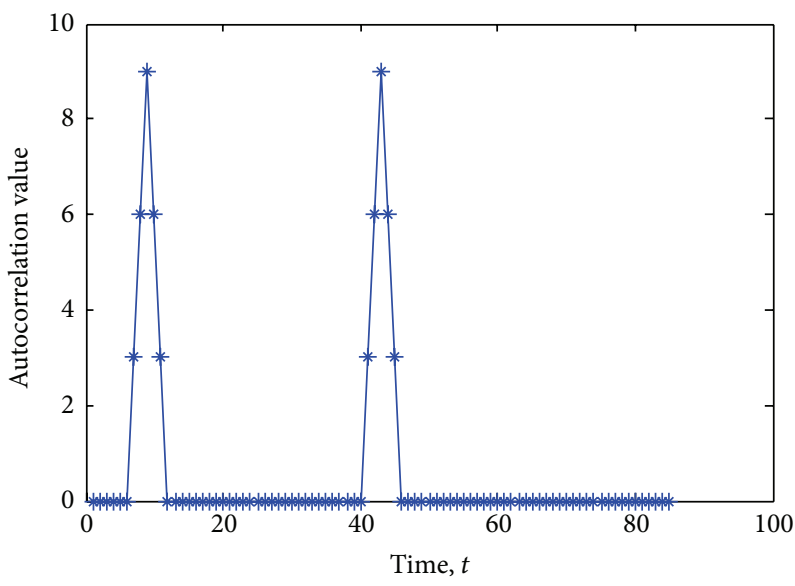

(c)

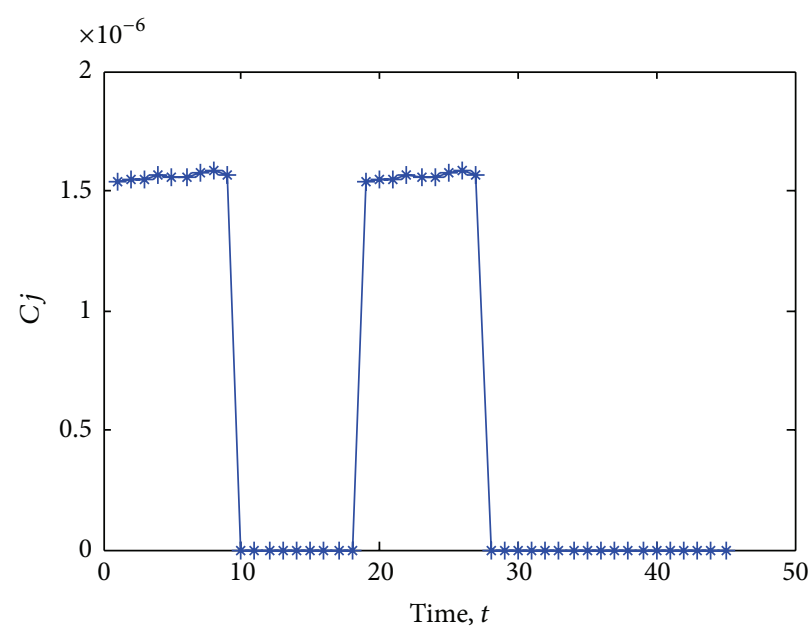

(b)

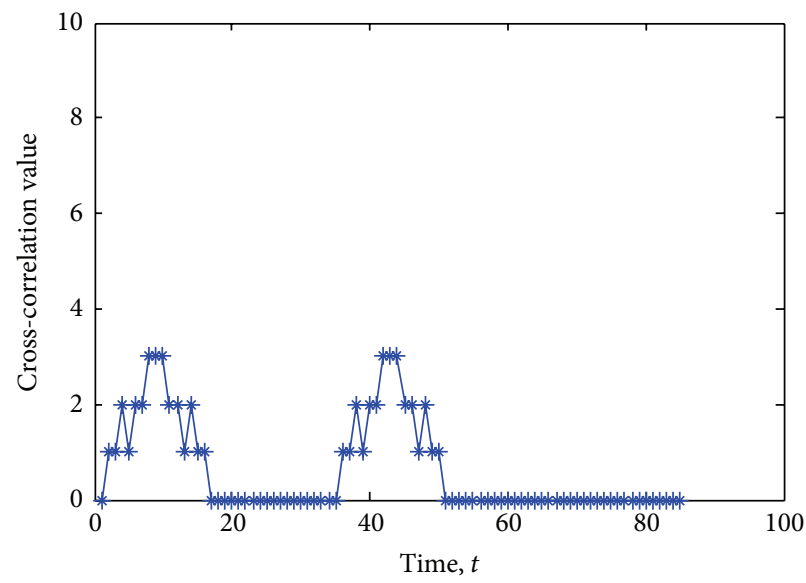

(d)

Figure 6: (a) Code word $Z_{0,1}$ of the code set of 3D optical code over GF(3). (b) Code word $Z_{2,2}$ of the code set of 3D optical code over GF(3). (c) Autocorrelation function of optical code $Z_{0,1}$ for the data bit stream 10100. (d) Cross-correlation function of optical codes $Z_{0,1}$ and $Z_{2,2}$, for the data bit stream 10100.

respectively. In (8a) and (8b) the correlation property for a pair of prime code words $C_{k}$ and $B_{k}$ with discrete data stream format are calculated.

$$
\begin{aligned}
& \lambda_{a}=\sum_{k=0}^{P-1} \sum_{m=0}^{P-1} \sum_{n=0}^{P-1} C_{k, m, n} C_{k \cdot m \cdot n \oplus \tau}=P^{2} \quad \text { for } 0 \leq \tau \geq P^{2}, \\
& \lambda_{c}=\sum_{k=0}^{P-1} \sum_{m=0}^{P-1} \sum_{n=0}^{P-1} C_{k, m, n} B_{k \cdot m \cdot n \oplus \tau} \leq 3 \quad \text { for } 0 \leq \tau \geq P^{2},
\end{aligned}
$$

where $C_{k, m, n}$ and $B_{k, m, n} \in(0,1)$ are an element of matrix $Z$, $P$ is the prime number over Galois field, and $\oplus$ denotes the modulo addition.

It can be observed from the above correlation function that the peak autocorrelation is $P^{2}$ which occurs when $C_{k, m, n}=C_{k, m, n}$. Also, the cross-correlation value is " 3 " occurring at all synchronized time $T$ when $C_{k, m, n} \neq B_{k, m, n}$. Figure 6 shows the two code words $Z_{0,1}$ and $Z_{2,2}$ of the code set of wavelength/time/space spread modified $3 \mathrm{D}$ multicarrier codes over GF(3) and of the length 9 and weight
9. It also illustrates that the autocorrelation property of $3 \mathrm{D}$ code sequence $Z_{0,1}$ having maximum peak value equals 9 at all synchronized times when the sequence follows the data stream 10100. Similarly, the cross-correlation property of 3D code sequence $Z_{0,1}$ with $Z_{2,2}$ is equal to 3 at all synchronized time $T$ for the same data stream 10100 . Similarly, Figure 7 represents the code words $Z_{2,0}$ and $Z_{1,1}$ and their autocorrelation and cross-correlation property, respectively, for the input data stream 10100.

Yen and Chen carried out the study of three-dimension Optical Code Division Multiple Access for optical fiber sensor networks. For efficient communication using OCDMA system, it is preferred to maximize the autocorrelation peak and minimize the cross-correlation function for sorting the correct signal and interference [18]. J. Singh and M. L. Singh present a new family of 3D wavelength/time/space codes named Golomb ruler-with-zero-insertions balanced codes for differential detection (GRZI-BCDD) using the unique interpulse distance property of Golomb rulers. The 3D code based on GRZI-BCDD requires fiber ribbons that 


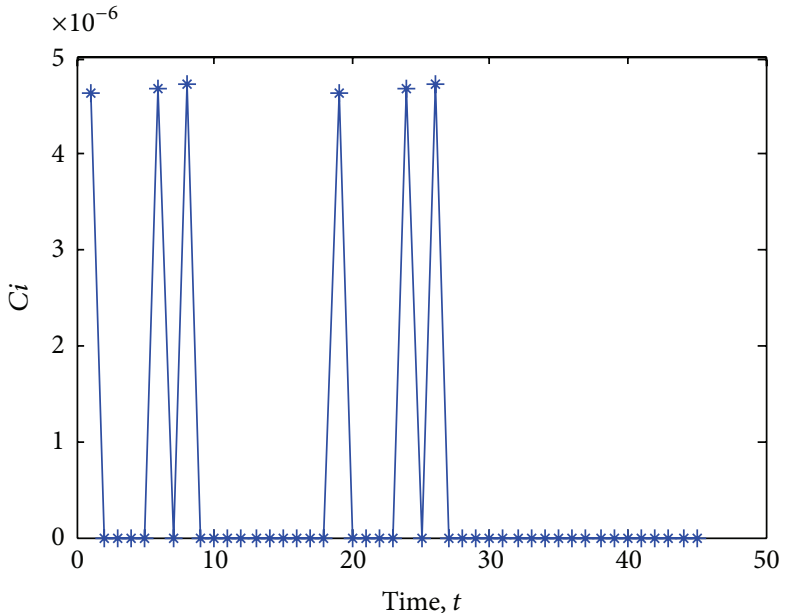

(a)

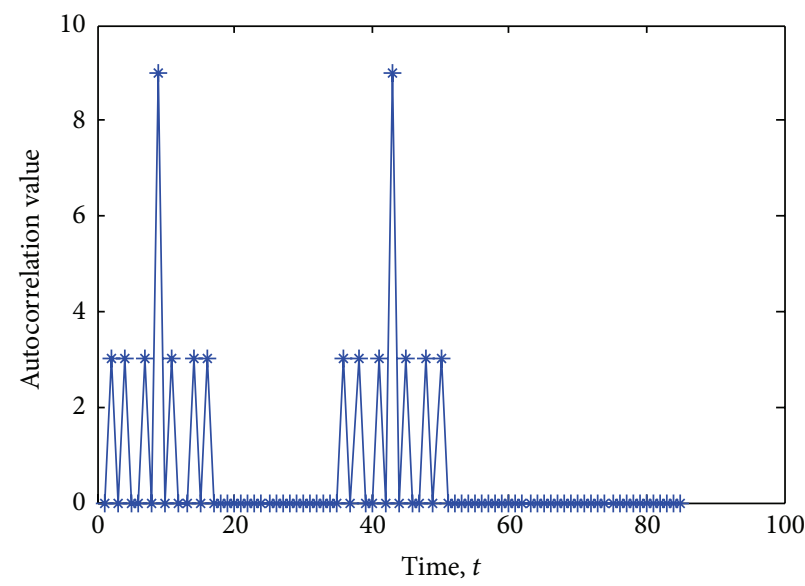

(c)

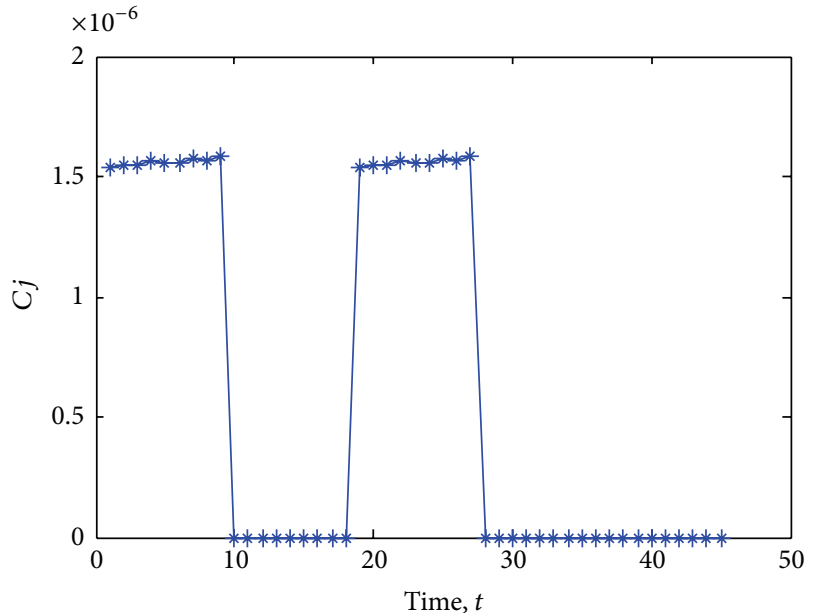

(b)

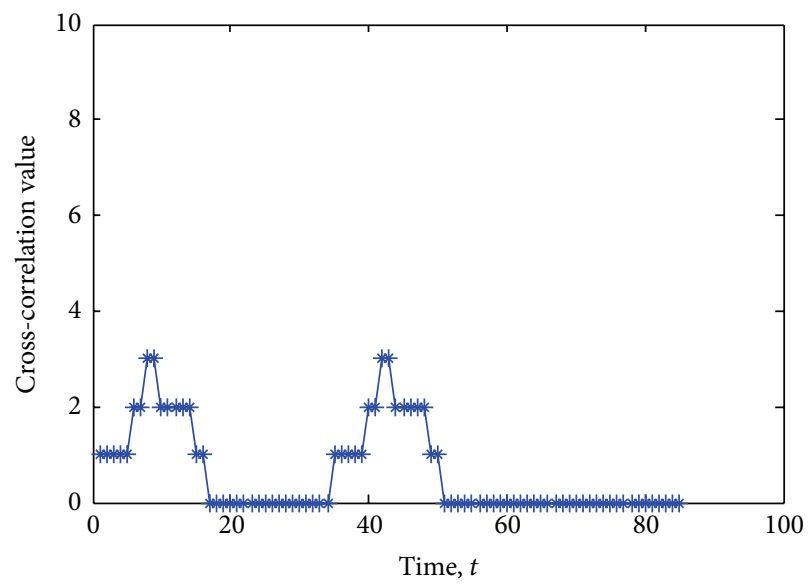

(d)

Figure 7: (a) Code word $Z_{2,0}$ of the code set of 3D optical code over GF(3). (b) Code word $Z_{1,1}$ of the code set of 3D optical code over GF(3). (c) Autocorrelation function of optical code $Z_{2,0}$ for the data bit stream 10100. (d) Cross-correlation function of optical codes $Z_{2,0}$ and $Z_{1,1}$, for the data bit stream 10100 .

increase the system complexity [19]. To improve the ability of suppressing the multiple access interference, the 3D code set must have a sufficient code length of each code word. The proposed code incorporates the third dimension to improve the code cardinality, security, and coding flexibility that will help in providing multimedia services and quality of services. The limiting factor is its implementation because the complexity of system hardware is increased as the dimension increases. However do not require fiber ribbons for practical implementation.

\section{Conclusion}

This work presents the proposed wavelength/time/space spread modified 3D multicarrier codes using the prime sequence algorithm. The code discussed in the text has been designed for the prime number $(P=3)$ having code length 9; however, it can be increased for the assumed prime number. Thus, by introducing more dimensions, this coding approach not only overcomes the problem of a sufficient number of users for a given prime number but also makes the proposed code more secure and support quality of services. However, a marginal complexity is increased from an implementation point of view due to additional dimension as compared to $1 \mathrm{D}$ and $2 \mathrm{D}$ codes. In addition, the generation algorithm of code is simple and has optimum orthogonal properties. Therefore, it reduces the cost involved. The simulation results on MATLAB depict that these 3D optical codes have maximum autocorrelation peak $\left(\lambda_{a}=P^{2}\right)$ with cross-correlation value $\left(\lambda_{c}=3\right)$.

\section{Competing Interests}

The authors declare that they have no competing interests.

\section{References}

[1] A. Stok and E. H. Sargent, "The role of optical CDMA in access networks," IEEE Communications Magazine, vol. 40, no. 9, pp. 83-87, 2002.

[2] J. A. Salehi, "Emerging optical CDMA techniques and applications," International Journal of Optics and Photonics, vol. 1, no. 1, pp. 15-32, 2007. 
[3] G.-C. Yang and W. C. Kwong, "Performance analysis of optical CDMA with prime codes," Electronics Letters, vol. 31, no. 7, pp. 569-570, 1995.

[4] R. Yadav and G. Kaur, "Optical CDMA: technique, parameters and applications," in Proceedings of the 4th International Conference on Emerging Trends in Engineering \& Technology, pp. 9098, Kurukshetra, India, October 2013.

[5] S. Park, B. K. Kim, and B. W. Kim, "An OCDMA scheme to reduce multiple access interference and enhance performance for optical subscriber access networks," ETRI Journal, vol. 26, no. 1, pp. 13-20, 2004.

[6] P. R. Prucnal, M. A. Santoro, and T. R. Fan, "Spread spectrum fiber-optic local area network using optical processing," Journal of Lightwave Technology, vol. 4, no. 5, pp. 547-554, 1986.

[7] A. J. Mendez, R. M. Gagliardi, V. J. Hernandez, C. V. Bennett, and W. J. Lennon, "Design and performance analysis of wavelength/time (W/T) matrix codes for optical CDMA," Journal of Lightwave Technology, vol. 21, no. 11, pp. 2524-2533, 2003.

[8] K. Yu and N. Park, "Design of new family of two-dimensional wavelength-time spreading codes for optical code division multiple access networks," Electronics Letters, vol. 35, no. 10, pp. 830-831, 1999.

[9] R. M. H. Yim, L. R. Chen, and J. Bajcsy, "Design and performance of 2-D codes for wavelength-time optical CDMA," IEEE Photonics Technology Letters, vol. 14, no. 5, pp. 714-716, 2002.

[10] E. S. Shivaleela and T. Srinivas, "Construction of wavelength/time codes for fiber-optic CDMA networks," IEEE Journal on Selected Topics in Quantum Electronics, vol. 13, no. 5, pp. 1370-1377, 2007.

[11] R. Yadav and G. Kaur, "Optical CDMA codes: a review," in Proceedings of the 7th International Conference Advanced Computing and Communication Technologies, pp. 263-269, Panipat, India, 2013.

[12] J. E. McGeehan, S. M. R. Motaghian Nezam, P. Saghari et al., “3D time-wavelength-polarization OCDMA coding for increasing the number of users in OCDMA LANs," in Proceedings of the Optical Fiber Communication Conference (OFC '04), pp. 444446, February 2004.

[13] S. Amiralizadeh and K. Mehrany, "Analytical study of multiple access interference and beat noise in polarization-wavelengthtime optical CDMA systems," in Proceedings of the 18th International Conference on Telecommunications (ICT '11), pp. 206-210, IEEE, Ayia Napa, Cyprus, May 2011.

[14] M. Ravi Kumar, S. S. Pathak, and N. B. Chakrabarti, "Design and performance analysis of code families for multi-dimensional optical CDMA," IET Communications, vol. 3, no. 8, pp. 13111320, 2009.

[15] C.-F. Hong and G.-C. Yang, "Multicarrier FH codes for multicarrier FH-CDMA wireless systems," IEEE Transactions on Communications, vol. 48, no. 10, pp. 1626-1630, 2000.

[16] R. Yadav and G. Kaur, "Design and performance analysis of 1D, 2D and 3D prime sequence code family for optical CDMA network," Journal of Optics, 2016.

[17] G. C. Yang and W. C. Kwong, "Prime codes with applications to CDMA optical and wireless networks," in Prime Codes with Applications to CDMA Optical and Wireless Networks, pp. 43108, Artech House, Norwood, Mass, USA, 2002.

[18] C.-T. Yen and C.-M. Chen, "A study of three-dimensional optical code-division multiple-access for optical fiber sensor networks," Computers and Electrical Engineering, vol. 49, pp. 136-145, 2016.
[19] J. Singh and M. L. Singh, "Design of 3-D wavelength/time/space codes for asynchronous fiber-optic CDMA systems," IEEE Photonics Technology Letters, vol. 22, no. 3, pp. 131-133, 2010. 

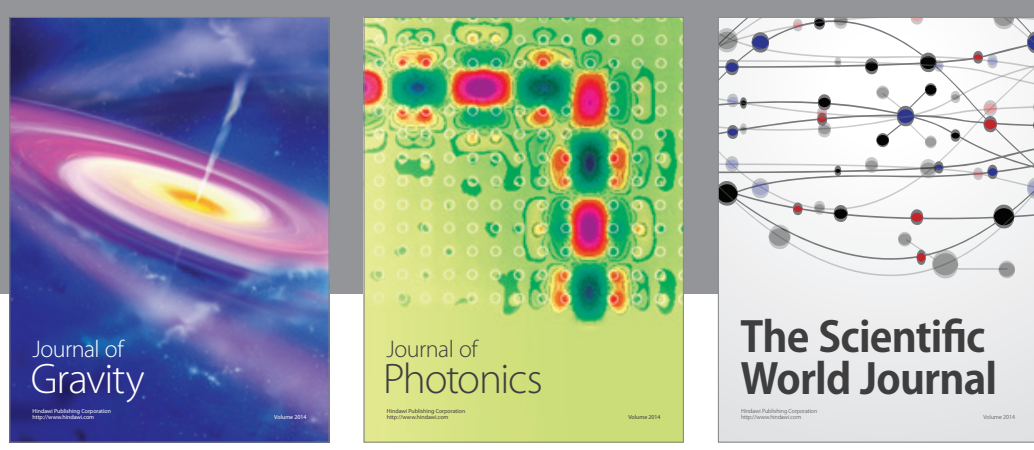

The Scientific World Journal
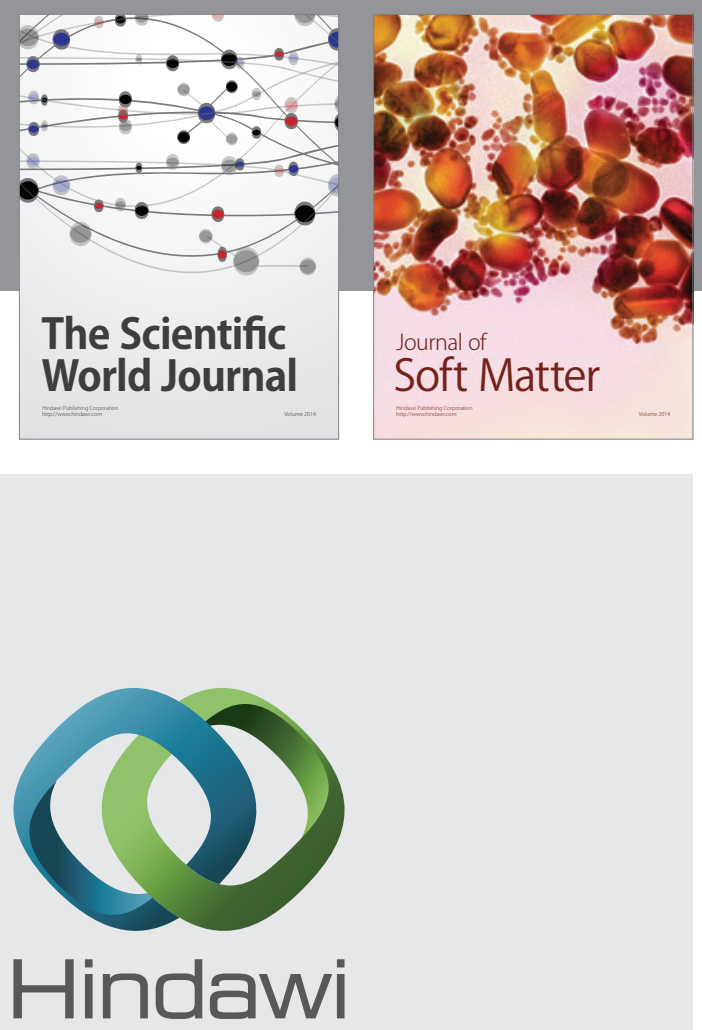

Submit your manuscripts at

http://www.hindawi.com

nternational Journal of

Statistical Mechanics
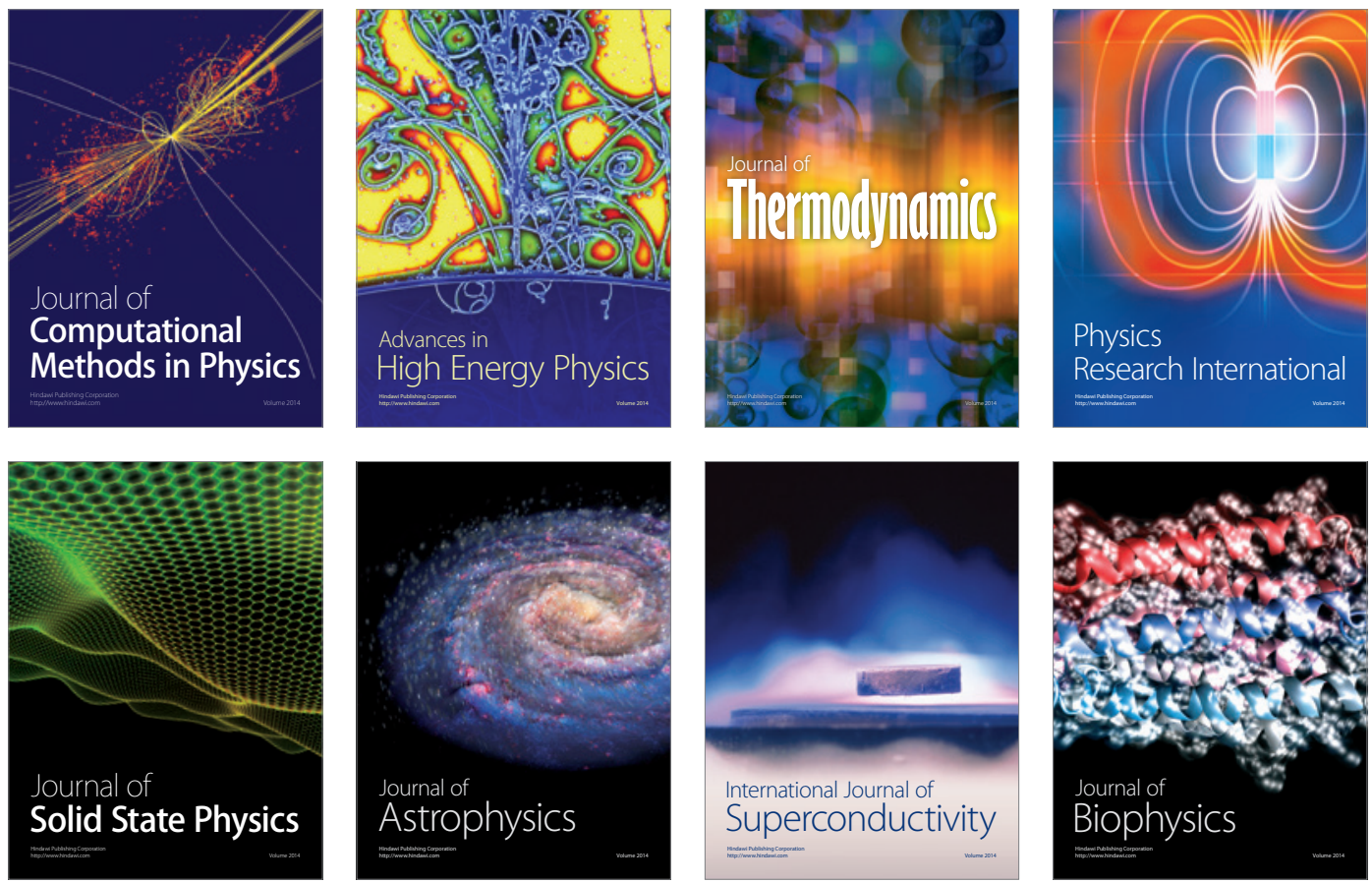
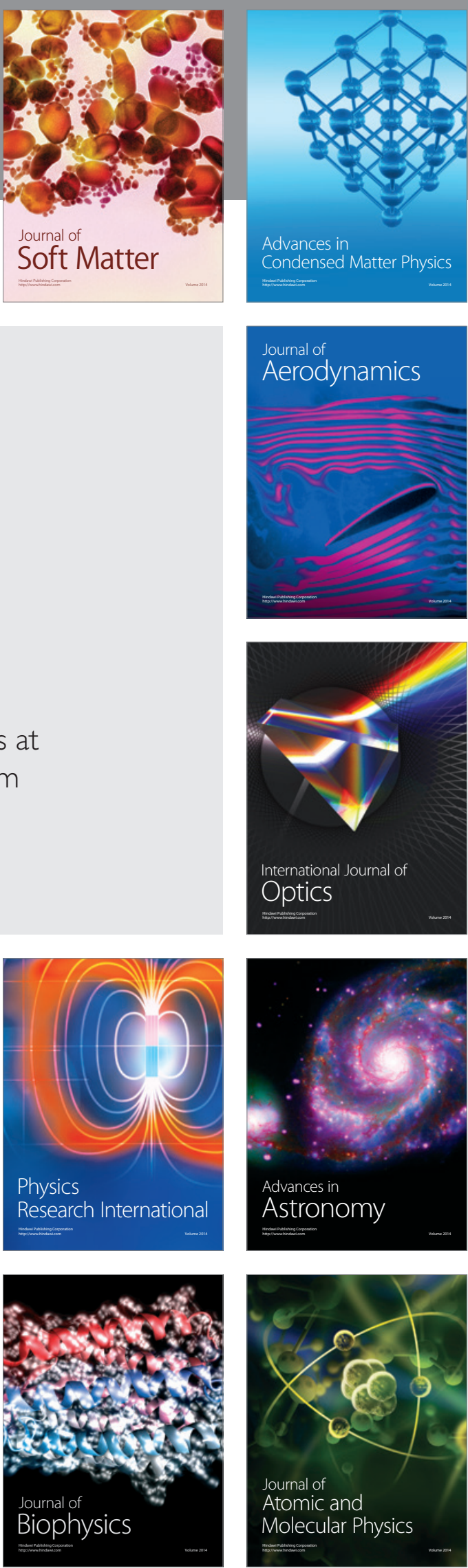